Pacific Journal of Mathematics

ABELIAN GROUPS IN WHICH EVERY ENDOMORPHISM IS A 


\title{
ABELIAN GROUPS IN WHICH EVERY ENDOMORPHISM IS A LEFT MULTIPLICATION
}

\author{
W. J. WICKLESS
}

Let $\langle G+\rangle$ be an abelian group. With each multiplication on $G$ (binary operation $*$ such that $\langle G+*\rangle$ is a ring) and each $g \in G$ is associated the endomorphism $g_{c}^{*}$ of left multiplication by $g$. Let $L(G)=\left\{g_{i}^{*} \mid g \in G, * \varepsilon\right.$ Mult $\left.G\right\}$. Abelian groups $G$ such that $L(G)=E(G)$ are studied. Such groups $G$ are characterized if $G$ is torsion, reduced algebraically compact, completely decomposable, or almost completely decomposable of rank two. A partial results is obtained for mixed groups.

Let $\langle G+\rangle$ be an abelian group. With each multiplication on $G$ (binary operation * such that $\langle G+*\rangle$ is a ring) and each $g \in G$ is associated the endomorphism $g_{l}^{*}$ of left multiplication by $g$ given by $g_{l}^{*}(x)=g * x, x \in G$. Let $L(G)$ be the set of all such endomorphisms, i.e., $L(G)=\left\{g_{l}^{*} \mid g \in G, * \varepsilon \operatorname{Mult}(G)\right\}$. In general all one can say is that $L(G)$ is a subset of the endomorphism ring $E(G)$. In this paper we consider abelian groups $G$ such that every endomorphism is a left multiplication.

DeFinition 1. An abelian group $G$ is multiplicatively faithful iff $L(G)=E(G)$.

We mostly follow the notations in [2]. Specifically: all groups are abelian, rings are not necessarily associative, $\otimes$ denotes the tensor product over $Z$ and $g \otimes$ - the natural map $x \rightarrow g \otimes x$ from $G$ into $G \otimes G, o(x)$ is the order of an element $x, Z(d)$ is the cyclic group of order $d$ and $Z(d)^{*}$ is the multiplicative group of units in $Z(d)$. For a prime $p$, we write $Z_{p}$ for the localization of $Z$ at $p$ and $\hat{Z}_{p}$ for the ring (or group) of $p$-adic integers. We use $t(A)[t(x)]$ for the type of a rank one torsion free group $A$ [element $x$ ] and $h(x)$ for the height sequence. Finally, $\langle S\rangle\left[\langle S\rangle_{*}\right.$ ] is the subgroup [pure subgroup] generated by $S$.

We begin by listing some simple results.

A. Let $\theta_{g}: \operatorname{Hom}(G \otimes G, G) \rightarrow E(G)$ be given by $\theta_{g}(\Delta)=\Delta \circ\left(g \otimes_{-}\right)$, $\Delta \in \operatorname{Hom}(G \otimes G, G), g \in G$. Then $G$ is multiplicatively faithful iff $\bigcup_{g \in G}$ Image $\theta_{g}=E(G)$.

Proof. Mult $G$, the group of all multiplications on $G$, is isomorphic 
to $\operatorname{Hom}(G \otimes G, G)$. Under this identification $\Delta \circ\left(g \otimes_{-}\right)=g_{l}$.

B. $G$ is multiplicatively faithful iff for each $\theta \in E(G)$, there exists $u \in G, \sigma \in$ Mult $G$ such that the following diagram commutes:

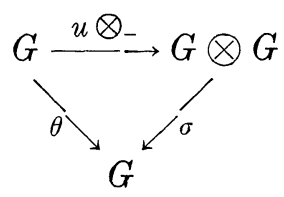

Proof. Obvious.

C. A divisible group is multiplicatively faithful iff it is torsion free. More generally, if $G=D \oplus R, D$ the maximal divisible subgroup of $G$ with $D$ torsion free, then $L(G)=E(G)$ iff $L(R)=E(R)$.

Proof. This follows directly from $(B)$ and elementary properties of the tensor product.

D. If $Z$ is a direct summand of $G$, then $L(G)=E(G)$. More generally, if $A$ is a ring, $1 \in A$, and $H$ is a unital $A$ module, then $A \oplus H$ is multiplicatively faithful.

Proof. Let $\theta \in E(A \oplus H)$. Set $u=1 \in A$, and define $\sigma \in \operatorname{Mult} G$ by $\sigma\left(\Sigma a_{i} \otimes x_{i} \oplus y\right)=\Sigma a_{i} \theta\left(x_{i}\right) ; \quad a_{i} \in A, \quad x_{i} \in A \oplus H, \quad y \in H \otimes(A \oplus H)$. Then

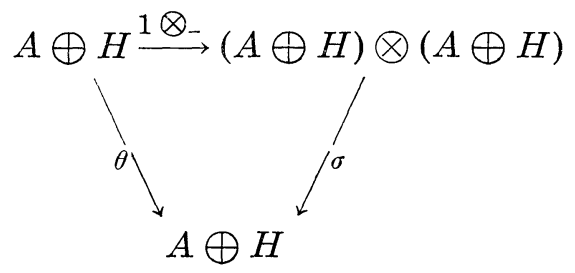

commutes.

E. Let $R(G)$ be the set of all right multiplications by elements of $G$ for all rings on $G$. Then $L(G)=E(G)$ iff $R(G)=E(G)$.

Proof. This follows from considering opposite rings.

Multiplicatively faithful torsion groups are easily characterized.

THeORem 1. Let $G$ be a torsion group. Then $G$ is multiplicatively faithful iff $G$ is bounded.

Proof. If $L(G)=E(G)$, then there exists $u \in G, \sigma \in$ Mult $G$ such that $\sigma \circ\left(u \otimes_{-}\right)=1_{G}$, where $1_{G}$ is the identity endomorphism. It follows 
that $n G=(0)$, where $n=o(u)$. If $n G=(0), n \in Z^{+}$, we can write $G=Z(n) \oplus H . \quad$ (D) applies to give $L(G)=E(G)$.

We next consider mixed groups, and characterize the multiplicatively faithful ones in one special case.

THEOREM 2. Let $G$ be mixed with maximal torsion subgroup $T=\bigoplus_{p} T_{p}$. Suppose that $T_{p} \neq(0)$ for only a finite number of primes $p$, and also that $G / T$ is homogeneous completely decomposable. Then $L(G)=E(G)$ iff (1) $G=T \oplus F$, (2) each rank 1 summand of $G / T$ has idempotent type, (3) $p(G / T)=G / T$ implies $T_{p}$ is bounded.

Proof. Suppose (1), (2) and (3) hold for $G$ as above. Let $T=$ $T_{1} \oplus T_{2}$, where $T_{1}$ is the sum of the bounded and $T_{2}$ the sum of the unbounded $p$ components of $T$. Since $T_{1}$ is bounded, write $T_{1}=Z(n) \oplus X$ with $X$ a unital $Z(n)$ module. $F \cong G / T$ is homogeneous, completely decomposable and nonzero. Say $F=A \oplus B$ where $A$ is torsion free of rank one and $B=\bigoplus_{\alpha \in I}(A)_{\alpha} . \quad(I=\varnothing$ is allowed. $)$ Since $t(A)$ is idempotent, $A$ is (may be regarded as) a subring with identity of $Q$ ([2], Th. 121.1). Moreover, since $p A=A$ only when $\left(T_{2}\right)_{p}=(0)$, $B \oplus T_{2}$ may be made into a unital $A$ module in the natural way. Thus, $X \oplus B \oplus T_{2}$ is a unital $Z(n) \oplus A$ module and (D) applies to show $G$ is multiplicatively faithful.

Conversely, let $L(G)=E(G)$ for $G$ satisfying the conditions of our theorem. Let $u \in G$ be such that $u_{l}^{*}=1_{G}$, * some multiplication on $G$. If $u \in p G$, clearly $T_{p}=(0)$.

Now consider a prime $p$ such that $u+T \in p(G / T)$. Since $(u+T)_{l}$ induces the identity endomorphism on $G / T$, it follows immediately that $u+T \in p^{n}(G / T)$ for all $n \in Z^{+}$. Write $u=p g+t=p g+t_{1}+t_{2}$, where $o\left(t_{1}\right)=p^{k},\left(o\left(t_{2}\right), p\right)=1$. If $t_{1}=0$, then $u \in p G$ and $T_{p}=(0)$. If $t_{1} \neq 0$, then, for all $x \in T_{p}$,

$$
x=u * x=\left(p g+t_{1}+t_{2}\right) * x=p(g * x)+t_{1} * x .
$$

(Since $\left(o\left(t_{2}\right), p\right)=1$ and $x \in T_{p}, \quad t_{2} * x=0$.) But $o[p(g * x)]<o(x)$, $o\left(t_{1} * x\right) \leqq o(x)$, so $o(x)=o\left(t_{1} * x\right) \leqq o\left(t_{1}\right)$. Thus $T_{p}$ is bounded.

Thus, for each $p$ such that $u+T \in p(G / T)$, we have $u+T \in p^{n}(G / T)$ for all $n \in Z^{+}$, and $T_{p}$ is bounded. Since $t(u+T)$ is the type of each rank 1 summand of $G / T$-(recall $G / T$ is homogeneous)-(2) and (3) hold. Let $T_{1}, T_{2}$ be as before. Since $T_{1}$ is bounded, $G=T_{1} \oplus H$ with $T_{2} \subseteq H$.

To establish (1), we must show that $T_{2}$ is a direct summand of $H$. Write $H / T_{2}$ as a direct sum of isomorphic rank one groups, $H / T_{2}=\bigoplus A_{i}$, and let $A_{i}=\left\langle a_{i}+T_{2}\right\rangle_{*}$ where $h\left(a_{i}+T_{2}\right)=\left(m_{i j}\right), m_{i j}=0$ or $\infty$ for all $i, j$. Since $p\left(H / T_{2}\right)=H / T_{2} \rightarrow\left(T_{2}\right)_{p}=(0)$, the following 
implication holds: $a_{i}+T_{2} \in p\left(H / T_{2}\right) \rightarrow a_{i} \in p H$. From this one easily obtains $H=T_{2} \oplus F$, where $F=\left\langle\left\{a_{i}\right\}\right\rangle_{*}$.

REMARK. The condition $T_{p} \neq(0)$ for only finitely many $p$ is necessary for the theorem. Let $G=\Pi_{p} Z(p)$. Then $T(G)=\bigoplus_{p} Z(p)$ is not a direct summand of $G$. However, $G / T(G)$ is homogeneous completely decomposable (torsion free divisible) and-as we shall see in Theorem $3-L(G)=E(G)$.

We next characterize reduced algebraically compact multiplicatively faithful groups. If $G$ is reduced algebraically compact, then $G=\Pi_{p} G_{p}$, where each $G_{p}$ is a complete module over $\hat{Z}_{p}$. Since each $G_{p}$ is fully invariant in $G\left(q G_{p}=G_{p}\right.$ for all $\left.q \neq p\right)$ and since Hom $\left(G_{p} \otimes G_{q}, G_{r}\right)=(0)$ unless $p=q=r$, it follows that $L(G)=E(G)$ iff $L\left(G_{p}\right)=E\left(G_{p}\right)$ for all $p$. Each $G_{p}$ may be written as a completion: $G_{p}=\left(B_{p}^{0} \oplus B_{p}\right)^{\wedge}$, where $B_{p}^{0}=\bigoplus_{\alpha \in I}\left(\hat{Z}_{p}\right)_{\alpha}, B_{p}=\bigoplus_{\beta \in J} Z\left(p^{k_{\beta}}\right), 0<k_{\beta}<\infty$. (See [2], § 40 for details.)

TheOREM 3. Let $G$ be reduced algebraically compact. Then $G$ is multiplicatively faithful iff, for each $p$, either $B_{p}^{0} \neq(0)$ or $G_{p}$ is bounded.

Proof. If $G_{p}$ is bounded, then $L\left(G_{p}\right)=E\left(G_{p}\right)$ by Theorem 1. If $B_{p}^{\circ} \neq(0)$, write $B_{p}^{\circ}=\hat{Z}_{p} \oplus B^{\prime}$. Then $G_{p}=\left(\hat{Z}_{p} \oplus B^{\prime} \oplus B_{p}\right)^{\wedge}$. Since $\hat{Z}_{p}$ is algebraically compact and pure in $G_{p}$ ([2], Th. 41.7, 41.9), we have $G_{p}=\hat{Z}_{p} \oplus G^{\prime}$. Since $G_{p}$ is a unital $\hat{Z}_{p}$ module, (D) gives $L\left(G_{p}\right)=E\left(G_{p}\right)$.

Conversely, suppose $G$ is reduced, algebraically compact and multiplicatively faithful. Then $L\left(G_{p}\right)=E\left(G_{p}\right)$ for all $p$. If for some $p B_{p}^{0}=(0)$, then $B_{p}=\bigoplus_{\beta \in J} Z\left(p^{k_{\beta}}\right) \subseteq T \subseteq G_{p} \cong \prod_{\beta \in J} Z\left(p^{k_{\beta}}\right)$, where $T$ is the torsion subgroup of the direct product. $\left(T \subseteq \hat{B}_{p}=G_{p}\right.$. $)$ Now, $G_{p} / T$ is torsion free divisible, thus homogeneous completely decomposable. Moreover, $T$ is a $p$-group, and $L\left(G_{p}\right)=E\left(G_{p}\right)$. Theorem 2 applies to give a splitting $G_{p}=T \oplus F$. Since $G_{p}=\hat{T}, F=(0)$. Thus, $G_{p}$ is a reduced algebraically compact torsion group, and is, therefore, bounded ([2], Cor. 40.3).

For the rest of the paper, we consider torsion free groups. First, we do the completely decomposable case.

TheOREM 4. Let $G=\bigoplus_{\lambda \in A} A_{\lambda}$, where each $A_{\lambda}$ is torsion free rank one. Then $L(G)=E(G)$ iff there exist subsets $\Lambda, \cdots, A_{n}$ of the index set $\Lambda$ and rank one groups $A_{\lambda_{1}}, \cdots, A_{\lambda_{n}}, \lambda_{i} \in \Lambda_{i}$, with (1) $\Lambda=$ $\bigcup_{i=1}^{n} \Lambda_{i}$ and (2) $t\left(A_{\lambda_{i}}\right)+t\left(A_{\lambda^{\prime}}\right) \leqq t\left(A_{\lambda^{\prime}}\right)$ for all $\lambda^{\prime} \in \Lambda_{i}, i=1, \cdots, n$.

Proof. Suppose $\Lambda_{1}, \cdots, A_{n} ; A_{\lambda_{1}}, \cdots, A_{\lambda_{n}}$ exist satisfying the above 
conditions. Without loss of generality, assume $\Lambda_{1}, \cdots, \Lambda_{n}$ are disjoint. Put $\lambda^{\prime}=\lambda_{i}$ in (2) to see that each $t\left(A_{\lambda_{i}}\right)$ is idempotent. Thus, each $A_{\lambda_{i}}$ can be made into a rank one ring with identity. Let $G_{i}=\bigoplus_{\lambda \in A_{i}} G_{\lambda}$. Due to (2), each $G_{i}$ can be regarded (in the natural way) as a unital $A_{\lambda_{i}}$ module. So we have $G=\bigoplus_{i=1}^{n} G_{i}$ is a unital $A$ module with $A=\bigoplus_{i=1}^{n} A_{\lambda_{i}}$ (ring direct sum). Since $A$ is a (group) direct summand of $G$, (D) applies.

Now suppose $G=\bigoplus_{\lambda \in A} A_{\lambda}$ with $L(G)=E(G)$. Choose $u \in G$, $\sigma \in$ Mult $G$ such that $\sigma \circ\left(u \bigotimes_{-}\right)=1_{G}$. Write $u=\sum_{i=1}^{n} a_{\lambda_{i}}, a_{\lambda_{i}} \in A_{\lambda_{i}}$. Then, for all $\lambda \in \Lambda, \pi \sigma\left(\bigoplus_{i=1}^{n} A_{\lambda_{i}} \otimes A_{\lambda}\right)=A_{\lambda}$, when $\pi$ is the projection from $G$ onto $A_{\lambda}$. Thus, for each $\lambda$, there exists at least one $i, 1 \leqq i \leqq n$, with $t\left(A_{\lambda_{i}} \otimes A_{\lambda}\right)=t\left(A_{\lambda_{i}}\right)+t\left(A_{\lambda}\right) \leqq t\left(A_{\lambda}\right)$. The desired partition of $\Lambda$ now easily can be constructed.

Let $G$ be an almost completely decomposable rank two torsion free group, i.e., $G \supseteqq A \oplus B \supseteqq d G$ for some $d \in Z^{+}$and rank one subgroups $A, B$ of $G$. We will obtain a numerical condition to show when such a $G$ is multiplicatively faithful. We may assume $t(A)$ and $t(B)$ are incomparable. (If $t(A)$ and $t(B)$ are comparable, then $G \cong A \oplus B$ by Theorem 9.6 of [1]. If $G \cong A \oplus B$, Theorem 4 gives a complete description of when $G$ is multiplicatively faithful.)

Let $A=\langle a\rangle_{*}, B=\langle b\rangle_{*}$ and let $d$ be the minimal positive integer with $d G \subseteq A \oplus B$. It is easy to show that $G=\langle A \oplus B, a+n b / d\rangle \subseteq$ $Q \oplus Q$ where $n$ is an integer with $(n, d)=1$. $(G / A \oplus B \cong Z(d)$.)

Let $h_{p}(x)$ be the $p$-component of the height sequence of $x$ and let $\Pi_{A}=\left\{p \mid h_{p}(a)=\infty\right\}, \Pi_{B}=\left\{p \mid h_{p}(b)=\infty\right\}$. It is also easy to show that $p \in \Pi_{A} \cup \Pi_{B} \rightarrow(p, d)=1$. Let $S$ be the multiplicative subgroup of $Z(d)^{*}$. generated by $\Pi_{A} \cup \Pi_{B}$.

Theorem 5. Let $G=\langle A \oplus B, a+n b / d\rangle$ be as above. Then $L(G)=$ $E(G)$ iff $t(A)$ and $t(B)$ are idempotent and $n \in S$.

Proof. Suppose $L(G)=E(G)$. If either $A$ or $B-A$ say-had nil type, then $A G=G A=(0)$ for any multiplication on $G$. (Recall that $t(A), t(B)$ are incomparable.) Thus, $1_{G}$ could not be represented as a left multiplication for any ring on $G$. Since $L(G)=E(G)$ we must have $t(A), t(B)$ idempotent.

Since $t(A), t(B)$ are idempotent we can assume, without loss of generality, that $h_{p}(a)=0, p \notin \Pi_{A}, h_{p}(b)=0, p \notin \Pi_{B}$. Choose $\sigma \in \operatorname{Mult}(G)$, $x=\alpha a+\beta b \in G, \alpha, \beta \in Q$, such that the following is a commutative diagram:

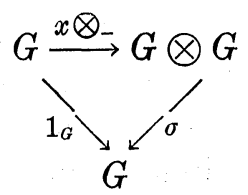


Let $\bar{\Pi}_{A}=\left\{m \in Z \mid m=p_{1}^{e_{1}} \cdots p_{k}^{e_{k}}, p_{i} \in \Pi_{A}\right\}$ and define $\bar{\Pi}_{B}$ similarly. Since $t(A), t(B)$ are incomparable, we have $\sigma(a \otimes b)=\sigma(b \otimes a)=0$; $\sigma(a \otimes a)=(c / h) a, h \in \bar{\Pi}_{A} ; \sigma(b \otimes b)=(e / k) b, k \in \bar{\Pi}_{B}$. Let $y=a+n b / d$. Then

$$
\sigma(y \otimes y)=\frac{1}{d^{2}}\left[\frac{c}{h} a+\frac{n^{2} e}{k} b\right] \in G .
$$

Since $d$ is relatively prime both to $n^{2}$ and to anything in $\bar{\Pi}_{A} \cup \bar{\Pi}_{B}$, we must have $c=c^{\prime} d, e=e^{\prime} d$

$$
\frac{1}{d}\left[\frac{c^{\prime}}{h} a+\frac{n^{2} e^{\prime}}{k} b\right] \in G .
$$

But $1 / d[a+n b] \in G . \quad$ A short computation yields: $n^{2} e^{\prime} / k-n c^{\prime} / h \equiv 0(d)$. Since $(n, d)=1$, we have $n e^{\prime} h-c^{\prime} k \equiv 0(d)$.

Now $\sigma[x \otimes a]=\sigma[(\alpha a+\beta b) \otimes a]=\alpha \sigma(a \otimes a)=\alpha\left(c^{\prime} d / h\right) a=$ $1_{G}(a)=a$, so $\alpha=h / c^{\prime} d$. Similarly, $\beta=k / e^{\prime} d$. Since $\alpha a+\beta b \in G$, we must have $c^{\prime} \in \bar{\Pi}_{A}, e^{\prime} \in \bar{\Pi}_{B}$. But then $n \equiv c^{\prime} k / e^{\prime} h(d)$, so $n \in S$. This shows the two conditions of our theorem are necessary for $L(G)=E(G)$.

Conversely, suppose $t(A), t(B)$ are idempotent and $n \in S$. Let $a, b$ be as before. Let $\lambda \in E(G)$. Since $t(A), t(B)$ are incomparable, $\lambda(a)=(m / h) a, \lambda(b)=(t / k) b ; h \in \bar{\Pi}_{A}, k \in \bar{\Pi}_{B}$. Now $\lambda(y)=1 / d[(m / h) a+$ $(n t / k) b] \in G$, so we must have $m k-t h \equiv 0(d)$.

Since $n \in S$, it is easy to choose $c, c_{1} \in \bar{\Pi}_{A}, e, e_{1} \in \bar{\Pi}_{B}$ such that $n e c_{1} \equiv c e_{1}(d)$.

Let $\sigma$ be defined by $\sigma(a \otimes a)=\left(d c / c_{1}\right) a, \sigma(b \otimes b)=\left(d e / e_{1}\right) b, \sigma(a \otimes b)=$ $\sigma(b \otimes a)=0$. To show $\sigma[G \otimes G] \subseteq G$, it is enough to check that $\sigma(y \otimes a), \sigma(a \otimes y), \sigma(y \otimes b), \sigma(b \otimes y)$ and $\sigma(y \otimes y)$ are all in $G$. All of these elements are obviously in $G$ except the last one, and

$$
\sigma(y \otimes y)=\frac{1}{d^{2}}\left[\frac{d c}{c_{1}} a+\frac{n^{2} d e}{e_{1}} b\right]=\frac{1}{d}\left[\frac{c}{c_{1}} a+n^{2} \frac{e}{e_{1}} b\right] .
$$

This is in $G$ iff $n\left(c / c_{1}\right) \equiv n^{2}\left(e / e_{1}\right)(d)$, which is true by choice $c, c_{1}, e, e_{1}$. Thus, $\sigma \in$ Mult $G$.

Now let

$$
g=\frac{1}{d}\left[\frac{c_{1} m}{h e} a+\frac{e_{1} t}{k e} b\right] .
$$

It follows directly that $\sigma \circ\left(g \mathbf{Q}_{-}\right)=\lambda$. (One need only check this identity on the independent set $\{a, b\}$.) It remains to show that $g \in G$. Now $g \in G$ iff $n\left[c_{1} m / h c\right] \equiv e_{1} t / k e(d)$. This congruence is easy to derive from $n e c_{1} \equiv c e_{1}(d)$ and $m k \equiv t h(d)$, both of which are given. Thus, $g \in G, g_{l}^{o}=\lambda$, and $G$ is multiplicatively faithful. 
The above theorem can be used to construct an example which shows that multiplicative faithfulness is not a quasi-isomorphism invariant for torsion free groups. Let $A=\left\{\left(m / 3^{k}\right) a \mid m, k \in Z\right\}$, $B=\left\{\left(m /(11)^{k}\right) b \mid m, k \in Z\right\}$, and let $G=\langle A \oplus B, a+2 b / 61\rangle$. Then $\Pi_{A}=\{3\}, \Pi_{B}=\{11\}$ and $2 \notin\left\langle\Pi_{A} \cup \Pi_{B}\right\rangle \subseteq Z(61)^{*} . \quad G$ is not multiplicatively faithful by Theorem $5 . \quad A \oplus B$ is multiplicatively faithful by Theorem 4. $G$ is quasi-isomorphic to $A \oplus B$, since $G \supseteqq A \oplus B \supseteqq$ $61 G$.

We give a name to a common occurence for torsion free groups.

Definition 2. Let $p$ be a prime and $A$ a rank one subgroup of a torsion free group $G$. $A$ is called $p$-dense in $G$ iff $p(G / A)=G / A$ and $G$ is $p$-reduced.

Theorem 6. Let $A$ be p-dense in $G$ for some prime p. Let $0 \neq a \in A$ and let $\Delta, \Gamma \in$ Mult $G$ be such that $a_{l}^{\Delta}=a_{l}^{\Gamma}$. Then $\Delta=\Gamma$.

Proof. Since $A$ is $p$-dense, $\operatorname{Hom}(G / A \otimes G, G)=(0)$. But then also $\operatorname{Hom}(G /\langle a\rangle \otimes G, G)=(0)$, since $A /\langle a\rangle \otimes G$ is the torsion subgroup of $G \mid\langle a\rangle \otimes G$ and $G$ is torsion free.

The exact sequence: $0 \rightarrow G \stackrel{a \otimes_{-}}{\longrightarrow} G \otimes G \rightarrow G \mid\langle a\rangle \otimes G \rightarrow 0$ yields: $0 \rightarrow \operatorname{Hom}(G /\langle a\rangle \otimes G, G) \rightarrow \operatorname{Mult} G \stackrel{\theta}{\rightarrow} E(G)$, where $\theta$ is given by $\theta(\Delta)=$ $\Delta \circ(a \otimes-)=a_{l}^{A} \in E(G)$. Since $\operatorname{Hom}(G /\langle a\rangle \otimes G, G)=(0), \theta$ is $1-1$.

\section{REFERENCES}

1. R. A. Beaumont and R. S. Pierce, Torsion Free Groups of Rank Two, Amer. Math. Soc., Mem. 38, Amer. Math. Soc., Providence, R. I., 1961.

2. L. Fuchs, Infinite Abelian Groups, v. I-II, Academic Press, New York, 1970, 1973. Received October 3, 1975 and in revised form November 5, 1975.

The UNIVERSity of ConNeCticut 



\section{PACIFIC JOURNAL OF MATHEMATICS}

EDITORS

RICHARD ARENS (Managing Editor)

University of California

Los Angeles, California 90024

R. A. BEAUMONT

University of Washington

Seattle, Washington 98105
J. DugundJI

Department of Mathematics

University of Southern California

Los Angeles, California 90007

D. Gilbarg and J. Milgram

Stanford University

Stanford, California 94305

\section{ASSOCIATE EDITORS}

E. F. BECKENBACH

B. H. NeumanN

F. WOLF

K. YosHIDA

\section{SUPPORTING INSTITUTIONS}

UNIVERSITY OF BRITISH COLUMBIA

UNIVERSITY OF SOUTHERN CALIFORNIA

CALIFORNIA INSTITUTE OF TECHNOLOGY

UNIVERSITY OF CALIFORNIA

STANFORD UNIVERSITY

UNIVERSITY OF TOKYO

MONTANA STATE UNIVERSITY

UNIVERSITY OF UTAH

UNIVERSITY OF NEVADA

WASHINGTON STATE UNIVERSITY

NEW MEXICO STATE UNIVERSITY

UNIVERSITY OF WASHINGTON

OREGON STATE UNIVERSITY

UNIVERSITY OF OREGON

OSAKA UNIVERSITY

AMERICAN MATHEMATICAL SOCIETY
NAVAL WEAPONS CENTER

Printed in Japan by International Academic Printing Co., Ltd., Tokyo, Japan 


\section{Pacific Journal of Mathematics}

\section{Vol. 63, No. $1 \quad$ March, 1976}

Ralph Artino, Gevrey classes and hypoelliptic boundary value problems ....... 1

B. Aupetit, Caractérisation spectrale des algèbres de Banach commutatives .... 23

Leon Bernstein, Fundamental units and cycles in the period of real quadratic

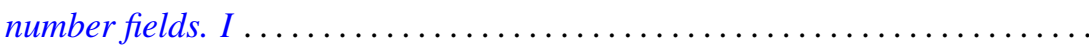

Leon Bernstein, Fundamental units and cycles in the period of real quadratic number fields. II.................................... 63

Robert F. Brown, Fixed points of automorphisms of compact Lie groups ........

Thomas Ashland Chapman, Concordances of noncompact Hilbert cube

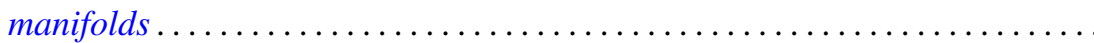

William C. Connett, V and Alan Schwartz, Weak type multipliers for Hankel

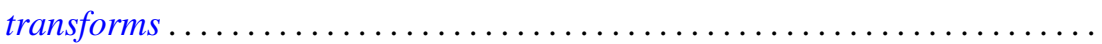

John Wayne Davenport, Multipliers on a Banach algebra with a bounded approximate identity .....................................

Gustave Adam Efroymson, Substitution in Nash functions ................ 137

John Sollion Hsia, Representations by spinor genera ..................

William George Kitto and Daniel Eliot Wulbert, Korovkin approximations in

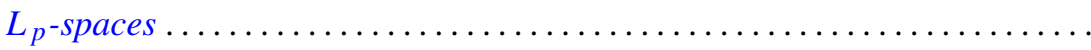

Eric P. Kronstadt, Interpolating sequences for functions satisfying a Lipschitz. condition ...........................................

Gary Douglas Jones and Samuel Murray Rankin, III, Oscillation properties of certain self-adjoint differential equations of the fourth order...

Takaŝi Kusano and Hiroshi Onose, Nonoscillation theorems for differential

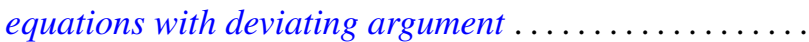

David C. Lantz, Preservation of local properties and chain conditions in commutative group rings. ...

Charles W. Neville, Banach spaces with a restricted Hahn-Banach extension property....

Norman Oler, Spaces of discrete subsets of a locally compact group ...

Robert Olin, Functional relationships between a subnormal operator and its minimal normal extension.

Thomas Thornton Read, Bounds and quantitative comparison theorems for nonoscillatory second order differential equations ...... .

Robert Horace Redfield, Archimedean and basic elements in completely distributive lattice-ordered groups...

Jeffery William Sanders, Weighted Sidon sets

Aaron R. Todd, Continuous linear images of pseudo-complete linear topological spaces.

J. Jerry Uhl, Jr., Norm attaining operators on $L^{1}[0,1]$ and the Radon-Nikodým property.

William Jennings Wickless, Abelian groups in which every endomorphism is a left multiplication. 